OS11 - 165

doi:10.1017/cjn.2016.341

\section{Brain Cancer Survival and Conditional Survival Rates in Canada (1992-2008)}

F. G. Davis ${ }^{l}$, Y. Yuan, Q. Shi, C. Nagamuthu, E. Andres, J. Ross

${ }^{1}$ University of Alberta, Edmonton, $A B$

faith.davis@ualberta.ca

To investigate patterns of survival and estimate conditional survival rates among brain cancer patients in Canada. METHODS: Canadian Cancer Registry data were obtained for all patients with primary brain cancer diagnosed between 1992 and 2008 $(\mathrm{n}=38,095)$. Follow-up ended with patient death or December 31, 2008, whichever occurred first. Crude Kaplan-Meier estimates were calculated at one, two, and five years post-diagnosis and also used to estimate conditional survival (restricted to 2000-2008). Age group, sex, residence and microscopic confirmation were considered in estimating rates for major histology types using multivariate models. RESULTS: The overall five-year survival rate was $27 \%$. Oligodendrogliomas had the highest 5-year survival rate (65\%, 95\% CI: 62.5-67.4\%) and glioblastomas the lowest $(4.0 \%$, 95\% CI: $3.7-4.3 \%$ ). Compared to Ontario, the age- and sexadjusted 5-year glioblastoma survival estimates were lower in British Columbia, Alberta and Manitoba-Saskatchewan, lower in all other regions for diffuse astrocytoma, and lower in ManitobaSaskatchewan for anaplastic astrocytomas. Estimates were significantly higher for oligodendrogliomas in Alberta, and for anaplastic oligodendrogliomas in Alberta and Quebec $(\mathrm{P}<0.05)$. Longer term conditional survival rates (surviving an additional 2 years 1-4 years after diagnosis) varied by histologic group. CONCLUSION: There is a need to further explore the underlying reasons for the observed variation in survival rates by region in an effort to improve the prognosis of brain cancer in the Canadian patient population. Conditional survival information has value for clinicians as they plan the course of treatment and follow-up for individual patients.

\section{0 - 1730 ORAL SESSION IV MENINGIOMA}

OS12 - 137

doi:10.1017/cjn.2016.342

The Geriatric Scoring System (GSS) for Risk Stratification in Meningioma Patients as a Predictor of Outcome in Patients Treated With Radiosurgery

O. Cohen-Inbar ${ }^{1,2,3}$

${ }^{I}$ Department of Neurological Surgery, Rambam Health Care

Center, Haifa Israel

${ }^{2}$ Molecular Immunology Laboratory, Technion Israel Institute of

Technology

${ }^{3}$ Department of Neurosurgery and Gamma-Knife Center,

University of Virginia, Charlottesville, Virginia

oc2f@virginia.edu

Meningiomas are the most common primary benign brain tumor. Radiosurgery (primary or adjuvant) allows excellent local control. The Geriatric scoring system (GSS) for pre-operative risk stratification and outcome prediction of patients with meningiomas has been previously reported. The GSS incorporates eight tumor and patient parameters on admission. A GSS score higher than 16 was previously reported to be associated with a more favorable outcome. We assessed the validity of the GSS score and its influence on outcome in patients treated with gamma-knife radiosurgery (GKRS). Methods: Patients treated with single session GKRS for WHO-1 meningioma during 1989-2013 at the University of Virginia were reviewed. A cohort of 323 patients, $50.2 \%(\mathrm{n}=162)$ males. Median age was $56(29-84)$, and median follow-up was 53.6 (6-235) months. Median tumor volume was 4.5 $\mathrm{cm} 3$ (0.2-23). Median margin and maximal doses were $15 \mathrm{~Gy}$ (836) and 32.3 Gy (20-65), respectively. Results: Tumor volume control was achieved in $87 \%(\mathrm{n}=281)$, and post-GKRS clinical neurological improvement reported in $66.3 \%(\mathrm{n}=214)$. The median change in KPS was +10 (range -30 to +40 ). The most common complication was intermittent headaches $(34.1 \%, \mathrm{n}=110)$ and cranial nerve deficits $(14.2 \%, \mathrm{n}=46)$. The GSS (calculated and grouped as GSS $>16$ and GSS <=16) was found to correlate with different Post-GKRS functional status $(\mathrm{p}<0.0001)$ and tumor control $(p=0.028)$. Conclusion: The GSS, used for risk stratification and outcome prediction in patients with meningiomas seems valid for patients undergoing single session GRKS. GSS score greater than 16 is associated with a better long-term functional status and tumor control.

\section{OS13 - 175}

doi:10.1017/cjn.2016.343

\section{Genetic Profiling of Radiation Induced Meningiomas}

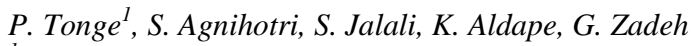

${ }^{I}$ Princess Margaret Cancer Centre, Toronto, ON

ptonge@uhnresearch.ca

One-third of all primary central nervous system tumors in adults are meningiomas, which arise from the meninges. Although the majority of meningioma cases are not associated with an environmental risk factor, it is well established that individuals receiving radiation treatment to the CNS are susceptible to radiation-induced meningiomas (RIMs). The genomic integrity of spontaneous meningiomas has been extensively profiled by whole genome sequencing and exome sequencing, providing a well-developed catalogue of meningioma associated mutations. In contrast, a comprehensive understanding of the molecular changes associated with RIMs is not available. Comparative genomic hybridization $(\mathrm{CGH})$ has previously revealed that $>90 \%$ of RIMs possess multiple regions of DNA copy number alterations, with the most common chromosomal loss being chromosome $1 \mathrm{p}$ and 22q. These CGH based studies of gross chromosomal alterations suggest that radiation induced meningiomas show no significant differences to spontaneous meningiomas. We have extensively characterized RIMs through the profiling of their global $\mathrm{CpG}$ methylation, gene expression and mutation signatures. The integration of these three platforms at base-pair resolution methylation has facilitated the identification of molecular changes that contribute to RIM. This is the largest cohort of profiled meningiomas to date, providing a robust characterization of unique RIM features that can be exploited for future therapies. 